\title{
The Influence of Magnetic Field on Annihilation of Positrons in Corroded Steel St-20
}

\author{
R. Pietrzak*, R. Szatanik and A. Jaworska \\ Institute of Physics, Opole University \\ Oleska 48, 45-052 Opole, Poland
}

\begin{abstract}
The positron lifetime in steel St-20 were determined before and following corrosion in $\mathrm{HCl}$ vapors. The influence of external magnetic field and remanent magnetization state on the parameters of the positron lifetime spectrum in the steel were investigated. It was found that the presence of magnetic field causes a decrease in the intensity of the component connected with annihilation of positrons in the close-to-the-surface lattice defects.
\end{abstract}

PACS numbers: 78.70.Bj, $61.72 \mathrm{Cc}, 82.45 . \mathrm{Bb}$

\section{Introduction}

For many years now the annihilation of positrons has found its application in research into kinetics and interaction of crystalline defects between one another and atoms of admixtures in solids. Numerous investigations proved that positrons lifetime depends on the shape and sizes of the defects and the intensity of the component of the spectrum of positron lifetime which is responsible for their annihilation in the defects is connected with their concentration [1-6].

Since 1999 we have carried on investigations of positron lifetimes emitted from the ${ }^{22} \mathrm{Na}$ source positrons in iron, nickel, and some steels with corroded surface [7-11]. We proved that the values of defect component of the positron lifetime spectrum and its intensity for samples with electrochemically corroded surface depend on the time of corroding, as well as on the chemical and mechanical conditions in which the corrosion was taking place. We also proved that after chemical removal of the corroded layer from the surface of the sample, the annihilation parameters of the positron lifetime spectra took values close to the annihilation parameters obtained for non-corroded samples.

In [12] we presented results of research on the influence of the initial surface defect state on annihilation characteristics of corroded steels St-29 and St-3S.

*corresponding author; e-mail: pietr@uni.opole.pl 
St-20 is a non-alloy surface-hardened steel often applied in conventional power plants. Very frequently installations built from this steel work in corrosive atmospheres in the presence of magnetic field. The present paper shows results of research into positron lifetime in steel St-20, with electrochemically corroded layer in the presence of an external magnetic field.

\section{Preparation of samples for investigation}

The annihilation of positrons was investigated in samples of St-20 before and after corroding them for 95 hours, the corrosion being induced by storing the samples in $\mathrm{HCl}$ vapors. A part of the samples corroded in the space between the poles of magnet in the field of the magnetic induction $B=267 \mathrm{mT}$ (Fig. 1). Following four groups of samples, which, prior to the measurements, were annealed

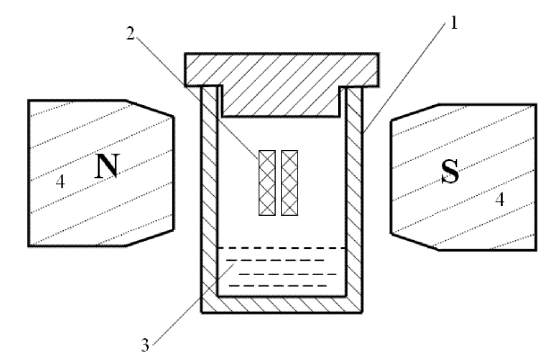

Fig. 1. Scheme of the apparatus used to investigate corrosion in $\mathrm{HCl}$ vapors in a magnetic field. 1 - plexiglass vessel; 2 - samples; 3 - $\mathrm{HCl}$ solution; 4 - poles of magnet.

under lowered pressure $\left(p<10^{-3}\right.$ mbar $)$ for 3 hours at $823 \mathrm{~K}$ and then cooled down to room temperature at $1 \mathrm{~K} / \mathrm{min}$. (bars A) were investigated:

- ones corroding in $\mathrm{HCl}$ vapors for $95 \mathrm{~h}$ outside a magnetic field (bars $\mathrm{B}$ ),

- ones corroding in $\mathrm{HCl}$ vapors for $95 \mathrm{~h}$ in a magnetic field, with induction $B=267 \mathrm{mT}$, perpendicular to the surface being subject to corroding and exposed to positrons in the course of investigations into annihilation (bars $\mathrm{C}$ ),

- ones stored prior to corroding in an external magnetic field for 100 hours, and then corroding in $\mathrm{HCl}$ vapors for $95 \mathrm{~h}$ outside the external magnetic field (bars D),

- ones stored prior to corroding in an external magnetic field for 100 hours, and then corroding in $\mathrm{HCl}$ vapors for $95 \mathrm{~h}$ in the external magnetic field (bars E).

The annihilation of positrons was investigated outside the magnetic field at room temperature. The registered spectra were subjected to deconvolusion into two or three components. In all of the cases in which the distribution of the spectrum was carried out into two components (further in the paper marked with indexes 1 and 2), the coefficient of adjustment of theoretical model to the 
experimental results $\left(\chi^{2}\right)$ was closer to one than in the cases of the distribution into three components. The results presented below relate to data obtained from the distribution of the spectra into two components.

\section{Results of measurements and their interpreting}

At the first stage of the investigation, the components of the spectrum of positron lifetimes were determined for each group of samples after their annealing at $823 \mathrm{~K}$ and slow cooling (bars A, Fig. 2). As it can be seen, the samples which were later subjected to successive stages of processing possessed the same annihilation characteristics within the limits of error $\left(\tau_{1}, \tau_{2}, I_{1}, I_{2}\right)$. The mean
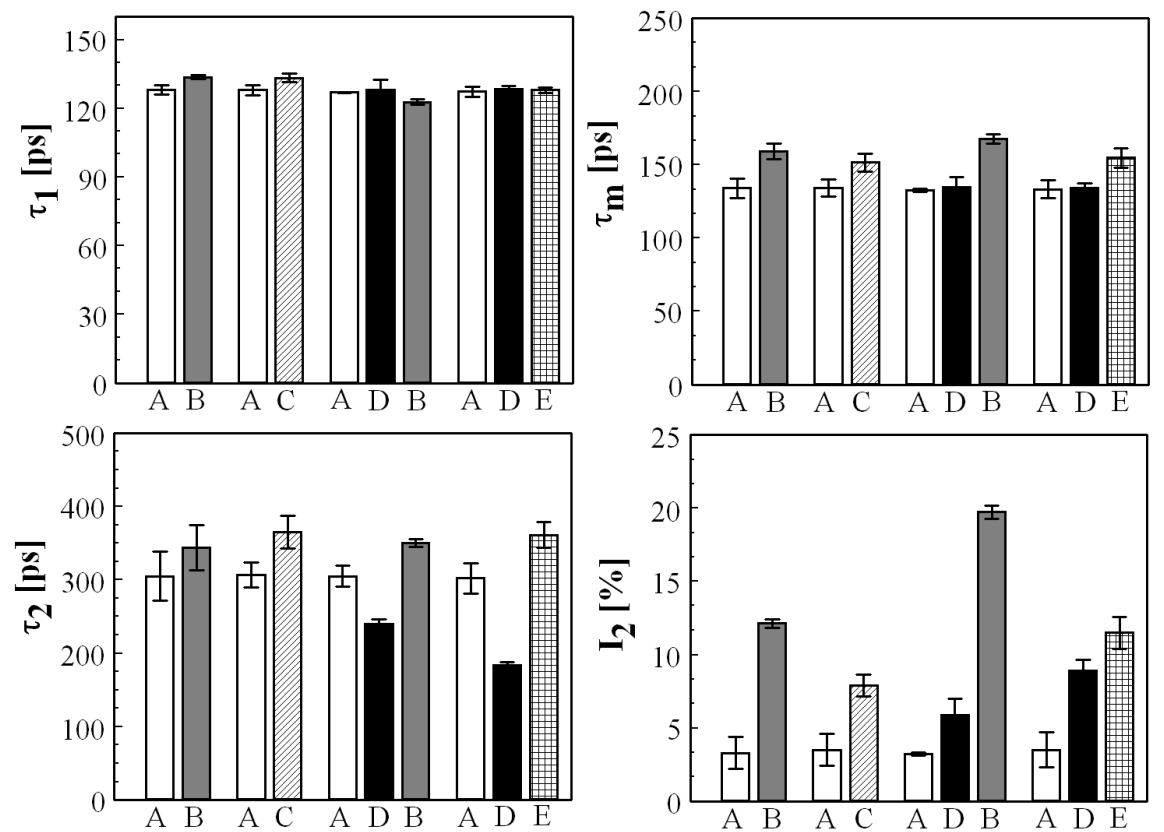

Fig. 2. Parameters of the spectra of positron lifetime in steel St-20 corroding in an external magnetic field and outside it - descriptions in the text.

positrons lifetime in the samples which were then corroded in $\mathrm{HCl}$ vapors was greater than before the corrosion (bars B). This was caused by the rise in the values of $\tau_{1}, \tau_{2}, I_{2}$. These results fully confirm the conclusions presented in [7-12] and prove that corrosion processes at the first stage of corroding cause an appearance and development of defects in the close-to-the-surface layer. The values of lifetimes $\tau_{1}$ and $\tau_{2}$ for the sample corroding in an external magnetic field are the same, within the limits of error, as for the samples corroding outside it. However, the intensity $I_{2}$ of the component of the spectrum, which is connected with annihilation of positrons in defects is markedly lower than for the samples corroding outside the 
external magnetic field (bars C). This seems to prove that magnetic field decreases the rate of the formation of close-to-the-surface defects.

The next two pairs of samples, prior to their placing in the corrosive atmosphere, were stored for 100 hours between the poles of magnet, in the geometry such as during corrosion in the external magnetic field. The aim of this procedure was to check whether the inner magnetizing of the samples, caused by storing them in an external magnetic field, alters the annihilation parameters of the investigated steel in relation to non-magnetized steel. It can be seen in Fig. 2 that the defect component of the spectrum of positrons lifetime $\left(\tau_{2}, I_{2}\right)$ in the steel kept in the external magnetic field does undergo a change. Its lifetime $\left(\tau_{2}\right)$ decreases, whereas the intensity $I_{2}$ rises in both types of samples (bar D and E). It is not possible, though, to univocally interpret the changes. The observed changes may be induced by internal magnetizing, but may as well result from corrosion of the samples surfaces in the atmospheric air, as the direction of the observed changes in the annihilation parameters is similar to that of the changes induced by corrosion in $\mathrm{HCl}$ vapors.

The type D samples, after being stored in an external magnetic field, corroded outside the field in $\mathrm{HCl}$ atmosphere during 95 hours, while the type $\mathrm{E}$ samples, following their storage in a magnetic field, corroded in similar chemical conditions, yet still in the magnetic field. It can be noticed (Fig. 2) that after corrosion of these types of samples, the component $\tau_{2}$ assumes, within the limits of error, the same value as for samples B and C. Like in the case of corroding, without the intermediate state, that is storing in a magnetic field, the intensity $I_{2}$ of the component of the spectrum relating to positrons annihilation in lattice defects is clearly greater than before the corrosion. Nevertheless, the intensity of the component $I_{2}$ for the samples kept in an external magnetic field and then corroding in this field, is markedly lower than that in the case when the corrosion was occurring outside the magnetic field. Investigations in the positron lifetime for this type of samples confirm the conclusion drawn from investigations into types $\mathrm{B}$ and $\mathrm{C}$ of samples that an external magnetic field decreases the rate of the formation of close-to-the-surface defects in the course of corrosion. The mechanism of this process is not yet fully understood and requires conducting additional research.

\section{Conclusions}

As it follows from the investigations carried out:

- positrons implanted into steel St-20 are heavily implanted in its corroded close-to-the-surface layer;

- annihilation parameters for steel corroding in an external magnetic field take values other than those when the corrosion was occurring outside the external magnetic field; 
- corrosion of steel St-20 in $\mathrm{HCl}$ vapors in the presence of magnetic field occurs more slowly than outside the field.

\section{References}

[1] W. Brandy, H.F. Wuang, Phys. Lett. 27, 700 (1968).

[2] J.K. Mc Kenzie, T.L. Khoo, A.B. Mc Donald, B.T. Mc Kee, Phys. Rev. Lett. 19, 146 (1976).

[3] M.J. Puska, R.M. Nieminen, J. Phys. F, Metal Phys. 13, 333 (1983).

[4] E. Kuramoto, H. Abe, M. Takenaka, F. Hori, Y. Kamimura, M. Kiura, K. Ueno, J. Nucl. Mater. 239, 54 (1996).

[5] Positron Spectroscopy of Solid State, Eds A. Dupasquier, A.J. Mills Jrs, IOS Press, Amsterdam 1995.

[6] P. Hautojarvi, Y. Johanson, T. Judin, P. Moser, M. Puska, A. Vehanen, J. Yli-Kaupilla, in: Proc 5th Int. Conf. on Positron Annihilation, Eds. R.R. Hasiguti, K. Fujiwara, Jpn. Inst. Met. Sendai 1979, p. 737.

[7] R. Pietrzak, R. Szatanik, M. Szuszkiewicz, Acta Phys. Pol. A 95, 647 (1999).

[8] R. Pietrzak, W. Smiatek, R. Szatanik, M. Szuszkiewicz, Fracture, Mechanics of Materiale and Structural Integrity 1, 178 (1999).

[9] R. Pietrzak, R. Szatanik, W. Smiatek, M. Szuszkiewicz, in: Proc. 31st Seminar on Positron Annihilation, September 1999, Jarnottówek (Poland), Eds. W. Świątkowski, K. Jerie, University of Opole, Opole 2000, p. 123.

[10] R. Pietrzak, R. Szatanik, W. Smiatek, in: Proc. 33rd Polish Seminar on Positron Annihilation, Turawa (Poland), Ed. K. Jerie, University of Opole, University of Wrocław, Opole 2001, p. 79.

[11] R. Pietrzak, in: Proc. 34th Polish Seminar on Positron Annihilation, Turawa (Poland), Ed. K. Jerie, University of Opole, University of Wrocław, Opole 2002, p. 61.

[12] J. Paja̧k, R. Pietrzak, M. Szuszkiewicz, Nukleonika 42, 185 (1997). 